\title{
The Cosmic Ray Nuclear Composition Measurement Performance of the Non-Imaging CHErenkov Array (NICHE)
}

\author{
John Krizmanic* \\ Universities Space Research Association \\ E-mail: jkrizmanic@usra.edu
}

\section{Douglas Bergman}

University of Utah

E-mail: bergman@physics . utah.edu

\section{Yoshiki Tsunesada}

Tokyo Institute of Technology

E-mail: tsunesada@cr.phys.titech.ac.jp

The Non-Imaging CHErenkov Array (NICHE) will eventually measure the flux and nuclear composition of cosmic rays from approximately $10^{15} \mathrm{eV}$ to $10^{18} \mathrm{eV}$ by using measurements of the amplitude and time-spread of the air-shower Cherenkov signal to achieve a robust event-byevent measurement of $X_{\max }$ and energy. NICHE will have sufficient area and angular acceptance to have significant overlap with TA/TALE, within which NICHE is located, in both TALE fluorescence and Cherenkov measurements allowing for energy cross-calibration. In order to quantify NICHE's ability to measure the cosmic ray nuclear composition, two different cosmic ray composition models, one based on the poly-gonato model of J. Hörandel [1] with an additional, extra-galactic proton component and the other based on the H4a model of T. Gaisser [2, 3], using simulated $X_{\max }$ distributions of the composite composition as a function of energy. These composition distributions were then unfolded into individual components via an analysis technique that included NICHE's simulated $X_{\max }$ and energy resolution performance as well as the effects of finite event statistics as a function of measured energy. In this paper, NICHE's ability to distinguish between these two CR composition evolution models and determine the individual components as a function of energy is presented.

The 34th International Cosmic Ray Conference,

30 July- 6 August, 2015

The Hague, The Netherlands

\footnotetext{
* Speaker.
} 


\section{NICHE Performance}

Co-sited with the Telescope Array Low-energy Extension (TALE) at the TA Middle Drum site, the NICHE experiment is designed to measure the cosmic ray flux and nuclear composition from $10^{15} \mathrm{eV}$ to $10^{18} \mathrm{eV}$ in its eventual complete deployment. In this paper, we evaluate the ability of NICHE to distinguish between two well-motivated models of cosmic ray nuclear composition evolution, one based on a poly-gonato model with an additional extra-galactic proton component [4] and one based on the H4a model [2, 3]. For the study presented in this paper, we use a 4component $(\mathrm{H}, \mathrm{He}, \mathrm{CNO}, \mathrm{Fe})$ version of each model.

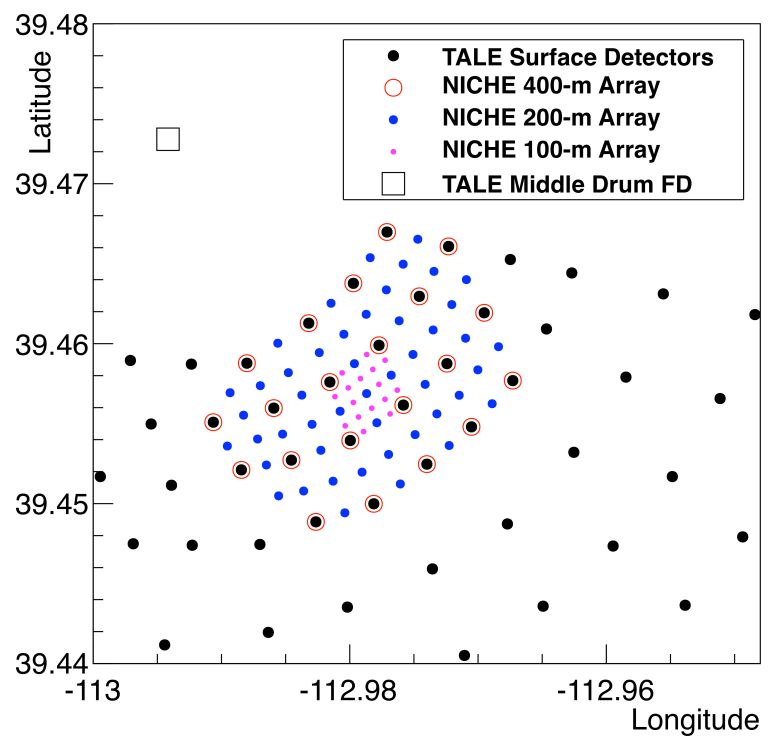

Figure 1: The layout of NICHE within TA/TALE used in this study: Middle Drum FD, open square; TALE SDs, black filled circles; NICHE 400-m array, large red open circles; NICHE 200-m array, blue filled circles; NICHE in-fill 100-m array, magenta filled circles.
NICHE will use a sparsely-filled array of easily deployable non-imaging Cherenkov detectors to measure the Cherenkov signal using both the Cherenkov Light Distribution (CLD) and the Cherenkov Time Width Distribution (CTWD) and thus determine the properties of cosmic ray induced air showers $[5,6]$. We have studied a variety of different NICHE array configurations, and for the results presented in this paper, we use a configuration of NICHE with 69 detectors with a separation of $200 \mathrm{~m}$ with an infill array of 25 detectors with a $100 \mathrm{~m}$ separation. The former allows for co-measuring air showers with TALE fluorescence measurements at higher energies (for cross-calibration) while the latter enhances NICHE's response to lower energy air showers. The use of TALE (imaging) Cherenkov measurements in combination with NICHE detectors is currently being studied [7]. The layout of NICHE used in the study presented in this paper is shown in Figure 1 where 400-m spaced detectors are highlighted since these could be used in

an initial deployment of a 21-counter array. Each NICHE detector uses a single 3-inch PMT coupled to a $45^{\circ}$ Winston cone yielding a $77 \mathrm{~cm}^{2}$ active area. A BG3 optical filter covers each PMT to constrain the wavelength range from $300 \mathrm{~nm}$ to $400 \mathrm{~nm}$.

The simulated response and resolutions of the NICHE array were determined using CORSIKA simulations. Cosmic ray events were generated in the range $10^{14} \rightarrow 10^{17.5} \mathrm{eV}$, using proton, helium, nitrogen, silicon and iron primaries. The detector array was modeled as a large, in both area and number of counters, array of $77-\mathrm{cm}^{2}$ detectors, with $50-\mathrm{m}$ spacing. This dense array was then decimated to provide sparse, large-spacing arrays of smaller areal size, including the layout used in this paper. In order to minimize simulation bias, no thinning was performed in the simulation for energies less than $10^{16} \mathrm{eV}$. For higher energies, showers were thinned, but the 
detector area was increased in such a way that the average weight of accepted photons was unity. Only photons arriving within $45^{\circ}$ of vertical were accepted to simulate the effect of the Winston cone. Atmospheric and quantum efficiency effects were handled in CORSIKA, leading to photoelectron $(\mathrm{PE})$ arrival times in any illuminated detectors.

After a 1-PE/ns random night sky background was included, the NICHE detector response was modeled using the measured response of a BLANCA PMT (with 9 ns risetime) followed by a second-order Butterworth filter of time scale $10 \mathrm{~ns}$. The result of the convolution was then sampled every $5 \mathrm{~ns}$ to simulate the operation of a FADC system, which will digitize the PMT signals. The simulated PMT signals were required to be $12-\sigma$ above the night-sky background for further consideration, simulating the L1 trigger. The L2 trigger was applied by requiring a 5 -fold coincidence of neighboring counters. In addition, the counter with the largest integrated signal was required not to be on the boundary of the array.

Analysis of the simulated events begins by finding the shower core by using the weighted average of counters that should contain the shower core using the NICHE counter with the largest signal and its largest-signal neighbors. The direction of the shower is then found by fitting the peak times in each counter, assuming a planar shower front. This provides a reasonably accurate estimate of shower direction that is needed to get the distance of each counter to the shower axis, $R_{P}$. The modeling of the counter time uncertainty imposes a $15-\mathrm{ns}$ counter-to-counter spread in absolute timing, based upon the uncorrected performance of commercial GPS hardware. With the direction estimate calculated, the shower core location is refined by requiring CLD measurements to be cylindrically symmetric and refining the core position to reduce the $\chi^{2}$ of the CLD fit. This procedure yields a shower core resolution of approximately $5 \mathrm{~m}$ and an angular resolution less than $0.5^{\circ}$, each defined by the peak of their respective distributions.

Each counter with $R_{P}>200 \mathrm{~m}$ provides

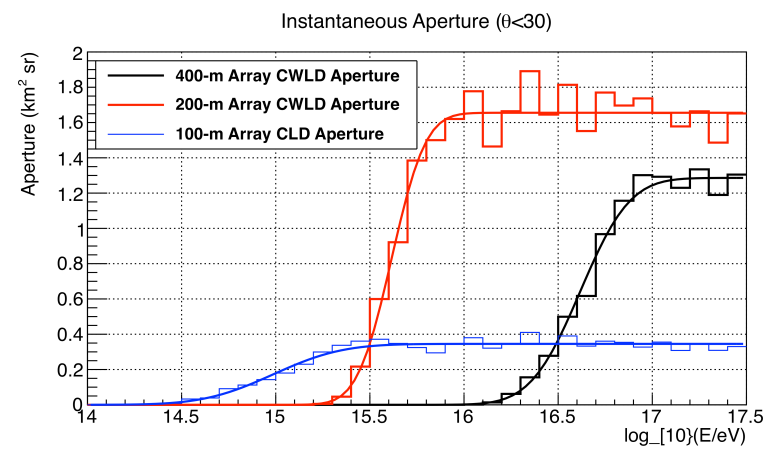

Figure 2: The reconstructed aperture for events with robustly reconstructed Xmax measurements and with $\theta<30^{\circ}$ for the 21 -counter 400 -m spacing array, the 69 - counter $200-\mathrm{m}$ spacing array and the 25 -counter, 100-m spacing in-fill array.

of NICHE as a function of energy can be quantified. These are respectively presented in Figures 2 , 3 , and 4 along with functional parameterizations obtained by fitting the appropriate data. Note that the figures illustrate the performance of each of NICHE's sub-arrays. The reconstructed resolution 


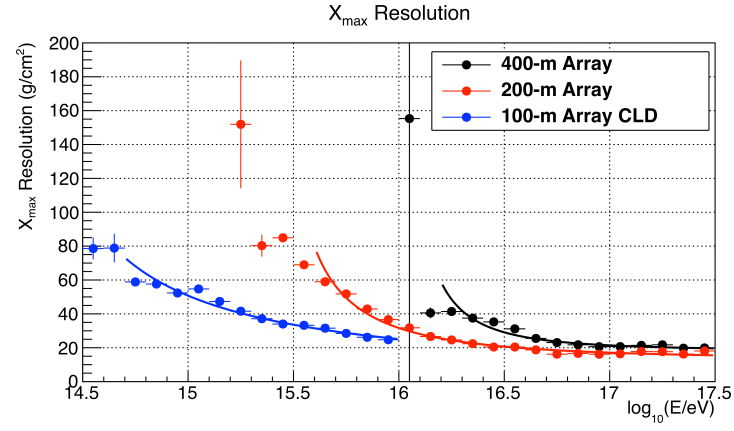

Figure 3: Reconstructed $X_{\max }$ resolution using the average measurements from individual counters, for a 21-counter 400-m spacing array and a 69-counter 200-m spacing array (using the CWTD), and a 25counter 100-m spacing in-fill array (using the CLD).

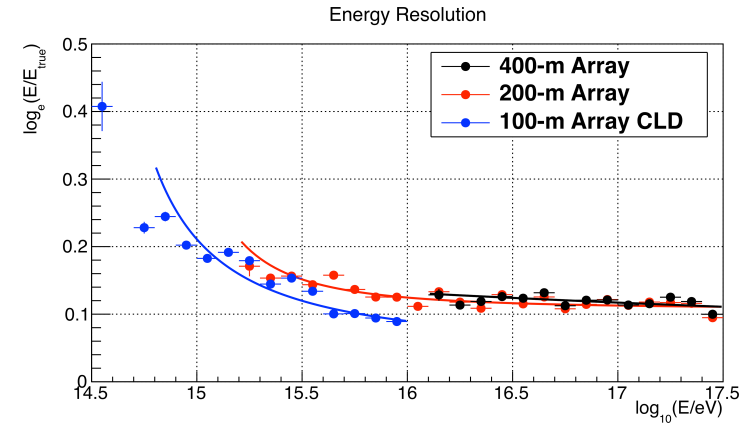

Figure 4: Reconstructed energy resolution using the average measurements from individual counters, for a 21-counter 400-m spacing array, a 69-counter 200-m spacing array, and a 25-counter 100-m spacing in-fill array (using the CLD).

includes equal amounts of protons, helium, nitrogen, silicon and iron primaries. For the study presented in this paper, the response of the 100-m infill array and the 200-m spaced array are employed. The 200-m array has better than $30 \mathrm{~g} / \mathrm{cm}^{2} X_{\max }$ resolution at energies above $10^{16} \mathrm{eV}$ and better than $20 \mathrm{~g} / \mathrm{cm}^{2}$ resolution above $10^{16.5} \mathrm{eV}$. The $100-\mathrm{m}$ in-fill array has better than 50 $\mathrm{g} / \mathrm{cm}^{2} X_{\max }$ resolution at energies above $10^{15} \mathrm{eV}$ and better than $30 \mathrm{~g} / \mathrm{cm}^{2}$ resolution above $10^{15.5}$ $\mathrm{eV}$. In terms of energy resolution, the $200-\mathrm{m}$ array has better than $20 \%$ resolution at energies above $10^{15.25} \mathrm{eV}$ while the $100-\mathrm{m}$ in-fill array has better than $20 \%$ resolution at energies above $10^{15.1} \mathrm{eV}$. Note that the individual counters have an additional $10 \%$ uncertainty added to total signal size to account for instrumental effects such as PMT calibration uncertainty.

\section{Cosmic Ray Composition Models}

In order to determine an experiment's CR composition measurement capability, physicallymotivated models are needed to quantify how the galactic cosmic ray composition evolves, and eventually is overtaken by an extra-galactic component. Two well-motivated models are used in the study presented in this paper.

The first model, denoted as PolyG4c, uses a 4-component version of the poly-gonato model detailed by J. Hörandel [1], by using the rigidity-based modeling of the spectral cutoff, compressing the the energy-dependent spectra for individual elements, from $z=1$ to $z=92$, into 4-components $(\mathrm{H}, \mathrm{He}, \mathrm{CNO}, \mathrm{Fe})$, and adding an extra-galactic (EG) proton component above $10^{16} \mathrm{eV}$. The EG proton component is defined to be that needed to yield an all-particle flux of $\phi(E)=3.0 \times 10^{24} E^{-3}$ particles $\cdot \mathrm{eV}^{-1} \cdot \mathrm{m}^{-2} \cdot \mathrm{s}^{-1} \cdot \mathrm{sr}^{-1}$. Details of the construction of the PolyG4c model are given in [4].

The second model, denoted as $\mathrm{H} 4 \mathrm{a} 4 \mathrm{c}$, is a 4-component version of the H4a model detailed by T. Gaisser in [2]. The H4a model in [2] is defined to have 5-components, $\mathrm{H}, \mathrm{He}, \mathrm{CNO}, \mathrm{MgSi}$, and Fe. To construct the 4-component $\mathrm{H} 4 \mathrm{a} 4 \mathrm{c}$ model, the fluxes of the $\mathrm{MgSi}$ and $\mathrm{Fe}$ components are combined to redefine the iron component. Figure 5 compares the prediction of $\langle\ln A\rangle$, where A is the atomic mass, versus energy of the two models. Note that model $\mathrm{H} 4 \mathrm{a} 4 \mathrm{c}$ predicts a heavier CR composition above $10^{17} \mathrm{eV}$ as compared to model PolyG4c. The simulated performance of 
NICHE to reconstruct each model is also shown in Figure 5, and the description of the CR nuclear composition reconstruction is given in the next section.

\section{NICHE CR Composition Determination}

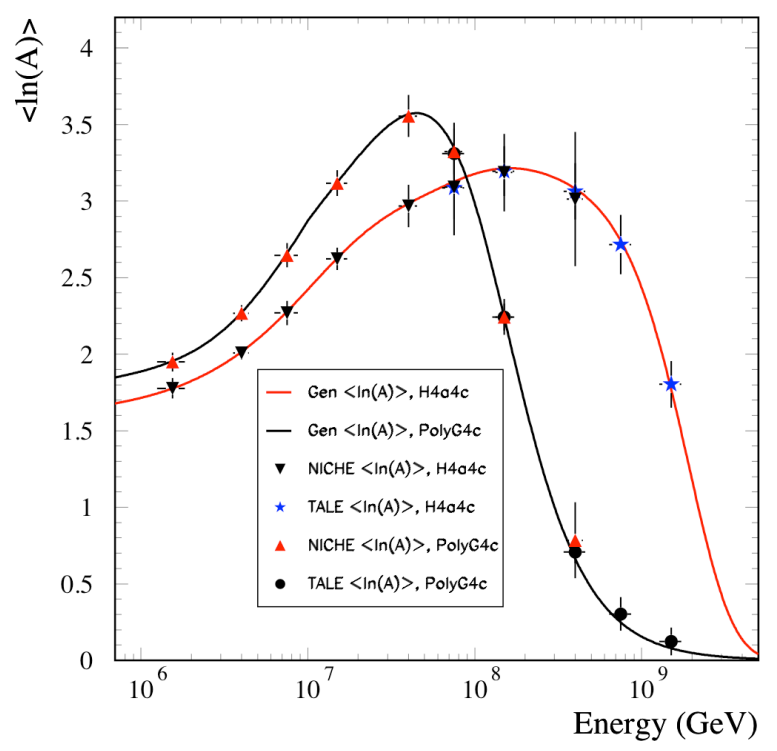

Figure 5: The generated and reconstructed $<\ln A>$ of the PolyG4c and H4a4c models.
One method for de-convolving distributions formed from multiple components is to use high-statistics individual distributions as templates that are then used in a multicomponent fitting procedure. The effects of instrument response, specifically $X_{\max }$ resolution, are incorporated both when the all-particle, composite $X_{\max }$ distribution is formed and for the individual component $X_{\max }$ templates themselves. A log-likelihood fitting procedure then can be employed to determine the composition of simulated or experimental data based upon Monte Carlo simulations of multiple components that form the composite distribution.

The procedure is now to employ this generation of model- and energy-dependent composite spectra and to unfold these to assess NICHE's capability to reconstruct the thrown composition for each model. As detailed in [4], an air shower simulation [8] was employed to generate high-statistics libraries of $X_{\max }$ distributions for proton, helium, nitrogen (denoted as CNO), and iron primary air showers in tenth of a decade energy bins. For each composition model, composite $X_{\max }$ distributions were formed based upon the energy-dependent component fractions in three bins per decade of energy from $10^{15}$ to $10^{17.8} \mathrm{eV}$ using NICHE's parameterized simulated response. The energy-dependent $X_{\max }$ resolution was included for each energy bin, based upon a parameterization of the $X_{\max }$ and energy resolution response, as shown in Figures 3 and 4. The statistics for each energy bin assumes two years of NICHE operation, using the reconstructed apertures (see Figure 2) and assuming a $10 \%$ duty cycle. The anticipated statistics $(N)$ of the composite distributions for each energy bin were Gaussian fluctuated with $\sigma=\sqrt{N}$. For the energy bin from $10^{17}$ to $10^{17.5} \mathrm{eV}$, this leads to all-particle statistics of approximately 1350 events in PolyG4c model and 1500 events in the H4a4c model for two years of NICHE operation. NICHE will be operational with TA/TALE, co-measuring air showers above $10^{16.8} \mathrm{eV}$, with TALE's event statistics surpassing NICHE's above $10^{17} \mathrm{eV}$. The TALE performance regarding CR composition measurement for each of the two CR composition evolution models is based upon using two-year statistics for TALE hybrid events based on [9] and assuming a $20 \mathrm{~g} / \mathrm{cm}^{2} X_{\max }$ resolution and a $15 \%$ energy resolution.

The composite distributions for the PolyG4c and H4a4c composition models were separately generated then unfolded assuming a 4-component ( $\mathrm{p}, \mathrm{He}, \mathrm{CNO}, \mathrm{Fe}$ ) model and assuming that 


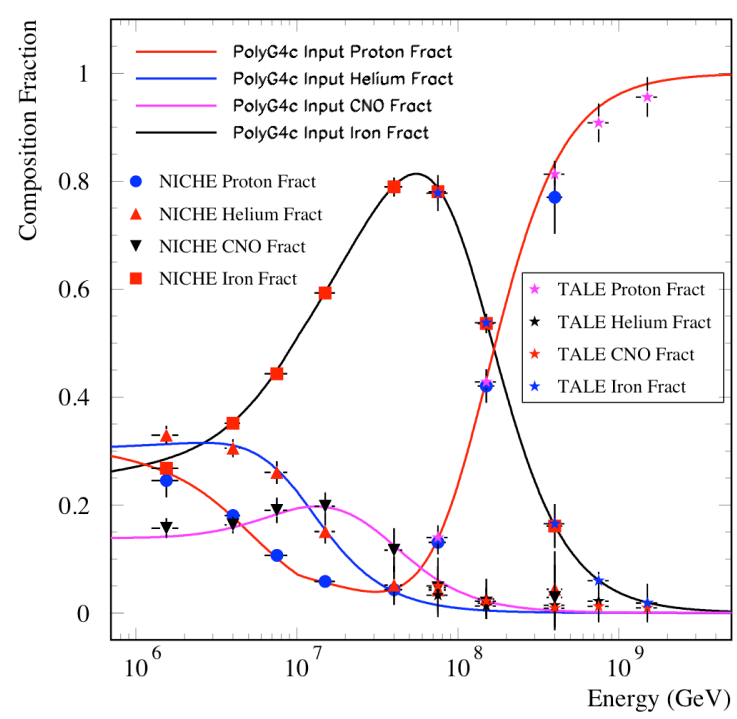

Figure 6: The generated and reconstructed fractions of events of a given nuclear species in model PolyG4c as a function of energy.

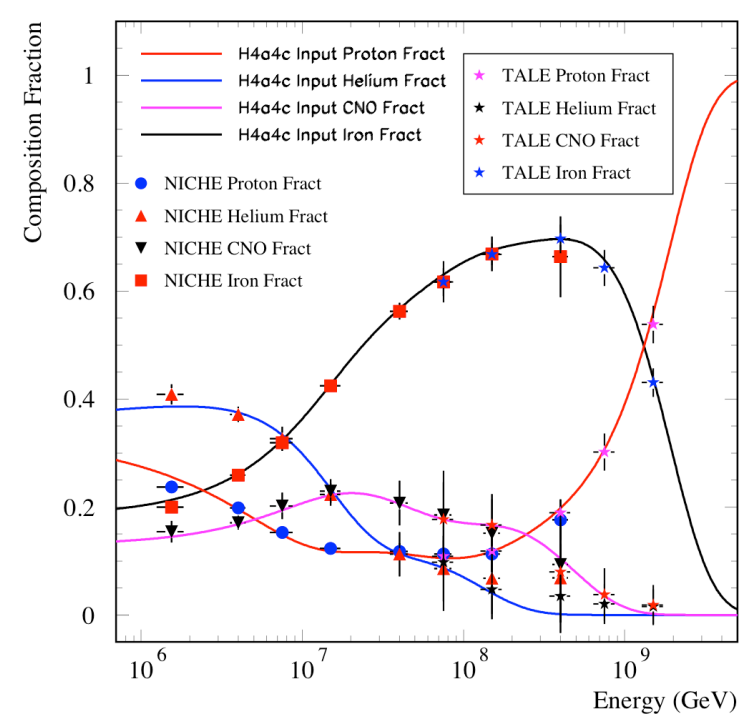

Figure 7: The generated and reconstructed fractions of events of a given nuclear species in model $\mathrm{H} 4 \mathrm{a} 4 \mathrm{c}$ as a function of energy.

the four component fractions added to unity. This unfolding yields the individual component fractions reconstructed by the analysis. This procedure was repeated 100 times, randomly choosing each $X_{\max }$ for each event from the appropriate library while also independently fluctuating the measurement statistics, yielding distributions of the unfolded fractions that are defined by a mean and an rms deviation, for each of the four components These then can be compared to the respective input 4-component fractions for each composition model.

The results in regards to reconstructing each of the two compositions are shown in Figures 6 and 7, illustrating NICHE's ability to accurately measure composition from $10^{15}$ to $10^{17.8} \mathrm{eV}$ based upon NICHE's simulated response. For a composition fraction of $\gtrsim 5 \%$, the simulated composition fraction determination has a resolution of $\lesssim 10 \%$ for both the PolyG4c and $\mathrm{H} 4 \mathrm{a} 4 \mathrm{c}$ composition models. For composition fractions $\lesssim 5 \%$, the unfolding procedure tends to over predict the reconstruction fractions, but by never more than a few percent. However, the dominant components are reconstructed with $\lesssim 10 \%$ resolution. The simulated results for the TALE CR composition determination demonstrates a similar trend. Figure 5 presents the results in terms of $<\ln A>$ as compared to the predictions of the two models.

In order to quantify the accuracy of the simulated NICHE to reconstruct the fractions of the two CR composition models, each of the four components was individually fit to the reconstructed data with an overall scaling parameter $\left(\alpha_{i}, i\right.$ represents either proton, helium, $\mathrm{CNO}$, or iron) as a free parameter. For data below $10^{17} \mathrm{eV}$ the results from the NICHE array were used while TALE results were used above $10^{17} \mathrm{eV}$. This yielded 10 data points for each of the four CR components. This was performed for each CR composition model and the results are summarized in Table 1. When the reconstructed fractions of the PolyG4c model were fit to the appropriate PolyG4c composition fractions, the scaling parameters for each component are fit to nearly unity with good $\chi^{2}$ values, which corresponds to nearly perfect agreement. Very similar results were obtained for the reconstructed fractions of the H4a4c model when fit with the respective H4a $4 \mathrm{c}$ 


\begin{tabular}{l|c|cc|cc}
\hline Component & Cosmic Ray & \multicolumn{2}{|l|}{ Simulated PolyG4c Results } & \multicolumn{2}{l}{ Simulated H4a4c Results } \\
\cline { 3 - 6 } Fitting Function & Component & Scale Factor & $\chi^{2}$ & Scale Factor & $\chi^{2}$ \\
\hline PolyG4c & Proton & $\mathbf{0 . 9 9} \pm \mathbf{0 . 0 2}$ & $\mathbf{2 . 4 9}$ & $0.71 \pm 0.01$ & 978 \\
PolyG4c & Helium & $\mathbf{1 . 0 1} \pm \mathbf{0 . 0 3}$ & $\mathbf{2 . 8 4}$ & $1.25 \pm 0.03$ & 9.89 \\
PolyG4c & CNO & $\mathbf{1 . 0 4} \pm \mathbf{0 . 0 6}$ & $\mathbf{1 . 1 1}$ & $1.13 \pm 0.05$ & 12.9 \\
PolyG4c & Iron & $\mathbf{0 . 9 9} \pm \mathbf{0 . 0 1}$ & $\mathbf{4 . 3 4}$ & $0.74 \pm 0.01$ & 1120 \\
PolyG4c & TOTAL & $\mathbf{1 . 0 1}$ & - & 0.96 & - \\
\hline H4a4c & Proton & $1.12 \pm 0.02$ & 1530 & $\mathbf{0 . 9 8} \pm \mathbf{0 . 0 2}$ & $\mathbf{8 . 4 4}$ \\
H4a4c & Helium & $0.81 \pm 0.03$ & 6.80 & $\mathbf{1 . 0 1} \pm \mathbf{0 . 0 3}$ & $\mathbf{2 . 9 6}$ \\
H4a4c & CNO & $0.78 \pm 0.05$ & 52.6 & $\mathbf{1 . 0 3} \pm \mathbf{0 . 0 5}$ & $\mathbf{0 . 6 8}$ \\
H4a4c & Iron & $0.78 \pm 0.01$ & 5005 & $\mathbf{0 . 9 9} \pm \mathbf{0 . 0 1}$ & $\mathbf{2 . 4 7}$ \\
H4a4c & TOTAL & 0.87 & - & $\mathbf{1 . 0 0}$ & - \\
\hline
\end{tabular}

Table 1: The results of fitting the simulated NICHE CR component reconstruction in ten energy bins between $10^{15} \mathrm{eV}$ and $10^{18.5} \mathrm{eV}$ to the individual CR component functions for the PolyG4c and H4a4c models with a scaling factor as the fit parameter. The TOTAL reported in the table is that obtained by adding the individual scale factors and dividing by four.

component functions, each with an independent scaling parameter. Table 1 also presents the results of fitting the reconstructed PolyG4c component fractions with the H4a4c component functions and vice versa. These results show that the fit scaling parameters are away from unity, and that the $\chi^{2}$ of the fit are much larger, especially for the proton and iron fractions. The results also show that the reconstructed helium fractions are fairly well fit regardless of which model's function are used, albeit with $\sim 25 \%$ error on the scaling factor. This may be due to the fact that the helium fraction predictions of the two models are similar in shape with respect to the reconstructed fractions once errors of the reconstructed fractions are considered.

\section{Discussion}

The results of the study presented in this paper demonstrates the ability of NICHE to accurately reconstruction the composition fractions of two different 4-component composition models in the energy range $10^{15} \rightarrow 10^{17.8} \mathrm{eV}$. The results also demonstrate similar performance for the simulated TALE response above $10^{16.8} \mathrm{eV}$. The decade overlap in energy scale between NICHE and TALE provides cross-calibration of CR flux measurements and nuclear composition determination. Detailed studies have also been performed using non-imaging Cherenkov detectors and TALE imaging-Cherenkov measurements to perform hybrid air shower reconstruction, and the results are reported at this conference in [7]. The use NICHE detectors and TALE imaging Cherenkov measurements to perform hybrid measurements provides a methodology of reducing the lower energy of the NICHE/TALE overlap to around $10^{15.5} \mathrm{eV}$, further strengthening the cross-calibration between NICHE and TALE. For the NICHE layout used in the study presented here, there exists another energy overlap in the decade above $10^{15.5} \mathrm{eV}$ using the CLD technique to reconstruct events with the infill array and using the CTWD technique to reconstruct events with the larger, 200-m spaced array. While the results are not detailed here, the simulated CR composition reconstruction 
using these two different techniques shows very good agreement over the entire energy overlap range and will provide an internal cross-calibration for NICHE.

\section{References}

[1] J. Hörandel, Astropart. Phys. 19, 193-220 (2003).

[2] T.K Gaisser, Astropart. Phys. 35, 801-806 (2012).

[3] T.K Gaisser, T. Stanev, S. Tilav, arXiv:1303.3565v1 (2013).

[4] J. Krizmanic, D. Bergman, P. Sokolsky, Proceed. 33rd ICRC (Rio de Janeiro), paper 0366 (2013).

[5] J. Krizmanic, D. Bergman, P. Sokolsky, Proceed. 33rd ICRC (Rio de Janeiro), paper 0365 (2013).

[6] D. Bergman, J. Krizmanic, Y, Tsunesada, Proceed. 34th ICRC (The Hague), paper PoS(ICRC2015)635 (2015).

[7] D. Bergman \& T. Abu-Zayyad, Proceed. 34th ICRC (The Hague), paper PoS(ICRC2015)345 (2015).

[8] P. Mikulski, Proceed. 26th ICRC (Salt Lake City) 1, 445-448 (1999).

[9] S. Ogio \& J. Matthews for the TA collaboration, Proceed. 33rd ICRC (Rio de Janeiro), paper 0717 (2013). 